\title{
Carta geomorfológica de la sección central y occidental de la Región Metropolitana de Santiago ${ }^{1}$.
}

\author{
María Victoria Soto Bäuerle \\ Departamento de Geografía, Universidad de Chile. mvsoto@uchile.cl \\ Carmen Paz Castro Correa \\ Departamento de Geografía, Universidad de Chile. cpcastro@uchile.cl \\ Giuliano Rodolfi \\ Dipartimento di Scienza del Suolo e Nutrizione della Pianta. Università di Firenze. \\ giuliano.rodolfi@unifi.it \\ Michael Märker \\ Universidad de Florencia, michael.maerker@unifi.it \\ Roberto Fernández Torres \\ Geógrafo, Escuela de Geografía, Universidad de Chile, robferdt@gmail.com \\ Rodrigo Padilla Torres \\ Geógrafo, Escuela de Geografía, Universidad de Chile. rpadilla@icono.dic.uchile.cl \\ Vanessa Rugiero de Souza \\ Geógrafa, Escuela de Geografía, Universidad de Chile, vanessarugiero@gmail.com
}

\section{RESUMEN}

Se presenta la carta geomorfológica de la mitad occidental de la Región Metropolitana de Santiago. En ella se definen unidades lito-morfológicas que facilitan el análisis de los sistemas de laderas, con el objeto de generar unidades o sistemas homogéneos de vertientes, en que la base de la interpretación morfodinámica se sustenta en la naturaleza litológica de los materiales. Estructuralmente, se destacan los relieves en estructuras monoclinales, resaltando la geología de los estratos y no la posición en el monoclinal, pues dado el ambiente de media y baja montaña, geodinámicamente, operarían de manera similar. En los relieves graníticos se distingue la presencia de superficies de erosión residual. Se incorpora también en las formas aluviales, la noción de terrazas según edad, distinguiendo entre pleistocénicas, holocénicas, e indeterminadas. Finalmente, como resultado se generan polígonos de unidades de similar morfogénesis, litología y geodinámica actual.

Palabras claves: Mapa geomorfológico sistemas de vertientes, sistemas depositacionales, estructura.

\begin{abstract}
The geomorphologic chart of the western part of the Santiago Metropolitan Region was made considering a lito-morphologic unit's definition purpose, especially for the interpretation and classification of homogenous slope systems. The chart goal was the identification of homogenous slopes systems, in which the base of the morpho dynamic interpretation is sustained in the geologic nature of the materials. Structurally, the monoclinals reliefs are emphasized. Also, the lithological nature of the layers and not it position in the monoclinal is considered into the analysis of the medium and low mountain environment because, from a geodynamic point of view, they generally operate in a similar way. In the intrusive relief's, remnants of erosional surfaces are distinguished. The notion of terraces is also considered in the alluvial forms according to age, distinguishing between pleistocénic, holocenic, and indeterminated terraces. As results, polygons of units of equal morphogenesis, lithologic and present geodynamics are generated.
\end{abstract}

Key words: Geomorphological map slope systems; depositional systems; structure. 
Carta geomorfológica de la sección central y occidental de la Región Metropolitana de Santiago

\section{INTRODUCCIÓN}

La taxonomía geomorfológica de ARAYAVERGARA $(1985,1988,1996)$ elaborada como base conceptual para el análisis de los ambientes de montaña y de fondo de valle asociado, y aplicada a la cuenca del río Mapocho, en la Región Metropolitana de Santiago, constituye la base teórica y metodológica de la presente investigación. Esta clasificación ha sido aplicada para Chile central, por MORALES (1988), WILLUMSEN (1989), FERRANDO (1994), CASTRO et al. (2002), MESINA (2003); SOTO y CASTRO (2003a, 2003b); CASTRO et al. (2003), SOTO et al., (2004), CHAVEZ (2005), SOTO et al. (2006). El énfasis de estos trabajos ha estado abocado principalmente a la aplicación de la taxonomía con las debidas adaptaciones, según las características especiales de las áreas estudiadas de alta, media y baja montaña.

En el contexto del análisis del sistema de fondo de depresión y flancos montañosos asociados, como es el caso de la Depresión de Santiago, se plantea la necesidad de establecer los grupos de formas y las relaciones genético-evolutivas del macizo granítico de la Cordillera de la Costa que circunscribe la sección sur y occidental del área de estudio.

Con base en la clasificación de vertientes de ARAYA-VERGARA (1985) para ambientes de media y baja montaña de Cordillera de la Costa, se estableció la importancia de la litología y la morfoestructura, toda vez que la morfodinámica de los relieves desarrollados en estructuras plegadas y la dinámica de las formas derivadas de rocas cristalinas es fundamental para la comprensión de los procesos geodinámicos de estos ambientes. La base conceptual de la carta geomorfológica se sustenta en las relaciones sistémicas de las clases de formas existentes y en las condiciones geodinámicas actuales de éstas.

Los aspectos genético-dinámicos fundamentales se sustentan en la relación vertientetalweg y sus relaciones con los sistemas de conos y glacis. Estas relaciones sistémicas y su relevancia para el análisis del espacio geográfico, se destacan en la taxonomía de ARAYA-VERGARA (1985), que considera en los ambientes de montañas, a las vertientes como sistemas y a las formas depositacionales basales, correlativos a éstos. Esta relación permite una concepción de la geodinámica actual del paisaje respecto de la acción de los agentes subaéreos.

Los procesos que se desarrollan en las formas de base de vertiente son indicativas de la dinámica de aporte de masa, actual y subactual (SOTO et al. 2006). Tales relaciones genético-evolutivas son claramente evidenciadas en ambientes de alta montaña andina, a través de los espesos depósitos de base de vertiente. En este contexto, destaca la relación directa entre frecuencia, espesor y dinámica de los depósitos coluviales y las vertientes de altura, la fuerte pendiente y la escasa o nula vegetación, relación favorecida por la marcada influencia de la acción de los relieves en estructuras plegadas.

Sin embargo, en los pisos inferiores de montaña, la relación vertiente-talweg y las acción de los agentes subáreos en las formas depositacionales de base de vertiente no son tan claramente evidenciadas. Por otra parte, la influencia de la tectónica y la morfoestructura es evidente en el modelado de las vertientes, donde los sistemas depositacionales se diferencian según la condición geoambiental, el substrato, el dominio morfoclimático de media y baja montaña, y la cobertura vegetacional (CASTRO et al. 2003).

Las relaciones de transferencia de masa en los ambientes de montaña y valle se llevan a cabo a través de los procesos de gravedad, como de los flujos de detritos, tanto desde las laderas de las cuencas, como en los propios cauces y conos aluviales asociados. Los sistemas de vertientes desarrollados, tanto en substratos rocosos estratificados de diferente resistencia como en relieves cristalinos, constituyen importantes centros de aporte de sedimentos heterométricos.

Los conos aluviales presentes en esta sección de la cuenca constituyen formas heredadas 
del Cuaternario, y no existen evidencias que permitan afirmar que pueda haber morfogénesis actual en ellas. Sin embargo, presentan una condición geodinámica actual de remodelado asociado a flujos y aportes de gravedad.

Consecuentemente, el propósito de esta investigación, es contribuir al conocimiento de los procesos geomorfológicos que ocurren en la sección central y occidental de la Región Metropolitana de Santiago. En este contex- to, se considera también como propósito la construcción de un modelo de clasificación de los hechos geomorfológicos sustentado en las formas y en el basamento geológico, tectónico y morfoestructural en que ellas han sido modeladas. La interpretación de los sistemas de vertientes como sistemas morfolitológicos y sus depósitos correlativos, se expresan en una cartografía a escala de menor detalle que la de ARAYA-VERGARA (1985), con un mayor nivel de generalización de las unidades del paisaje.

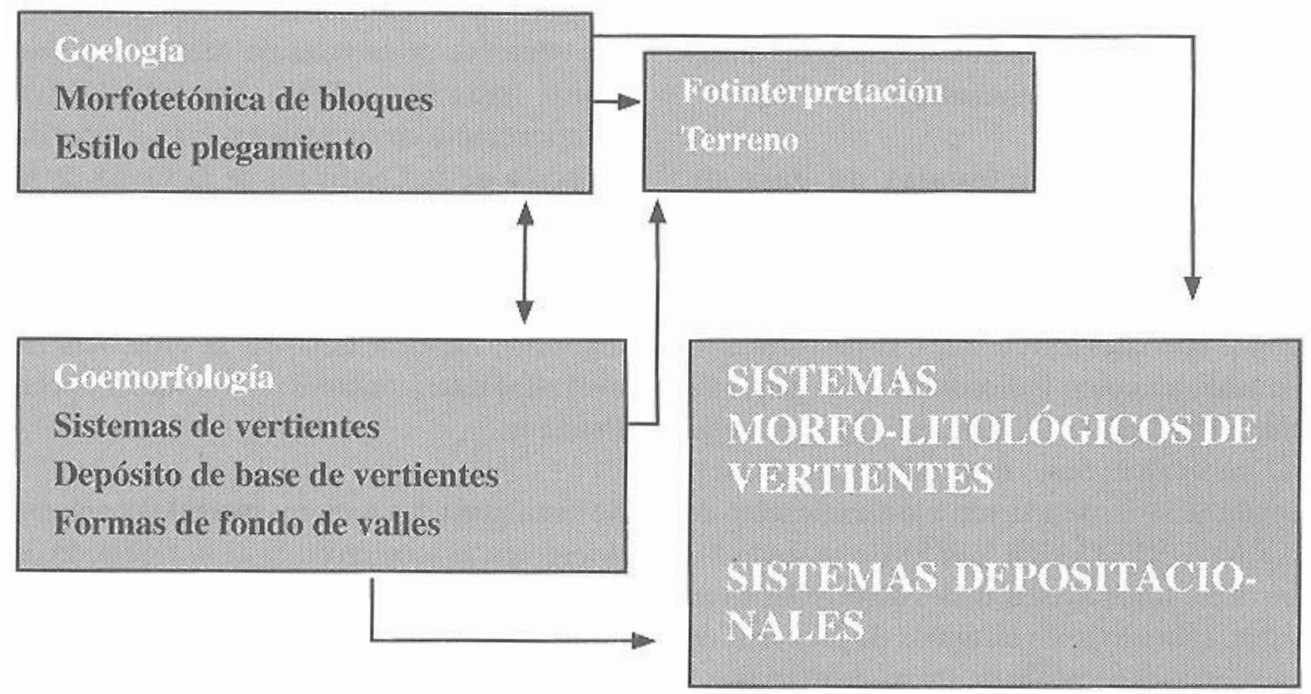

\section{MATERIALES Y MÉTODOS}

Para la realización de la carta geomorfológica del área de estudio, se siguió el siguiente esquema metodológico:

\section{Influencia Estructural}

Ha sido considerada como la variable que expresa una condición general del aporte potencial de masa desde las vertientes, hecho ligado a la exposición de los estratos rocosos que afloran en las formaciones plegadas, correspondientes en general a secuencias sedimentarias y volcánicas. Los relieves macizos corresponden principalmente a intrusivos granitoides. Se incluyó también las características del fallamiento, estilo de plegamiento, litología y edad según los antecedentes de WALL et al., (1996) y de SELLÉS y GANA (2001).

\section{Los sistemas de vertientes}

Al igual que en la taxonomía de ARAYAVERGARA (1985), se consideró de fundamental importancia el analizar las vertientes como sistemas, las que se tratan como unidades integrales de geología y procesos dinámicos actuales, así como evaluar su rol como aportadoras de masa, sobre todo en el caso de vertientes modeladas en rocas estratificadas 
94 Carta geomorfológica de la sección central y occidental de la Región Metropolitana de Santiago

con aflojamientos rocosos, desprovistas de vegetación y fuerte pendiente.

Según la clasificación de ARAYA-VERGARA (1985), el tratamiento para las áreas de baja y media montaña debiera estar referido a la acción de los agentes exógenos, es decir, según la tendencia erosiva. Sin embargo, ante la no menos importante cobertura espacial de formas estructurales en tales áreas, se ha considerado en este trabajo, necesario destacar este rasgo, pues ello expresa mejor la condición de aportadores potenciales de masa de estos sistemas de vertientes (SOTO et al 2006). La interpretación de estos procesos dinámicos deberá realizarse a la luz de los pisos altitudinales y las condiciones biogeográficas de las áreas a ser estudiadas.

\section{Formas depositacionales de base de vertientes.}

Corresponde a sistemas de conos, glacis y conos aluviales torrenciales. Estas formas se consideran como indicadoras de la geodinámica heredada del Cuaternario en los sistemas de vertientes. Son mapeadas y analizadas según génesis, de acuerdo a la clasificación de ARAYA-VERGARA (1985), destacando los procesos dinámicos actuales desarrollados en éstas, sobre todo en términos de procesos de erosión y generación de flujos de detritos.
Formas depositacionales de fondo de depresión.

Corresponden a los grandes conos regulares del río Maipo y Mapocho y las superficies de terraplenamiento fluvial y palustre desarrollados durante el Cuaternario. Estas formas han sido incorporadas según la clasificación de ARAYA-VERGARA (1985).

\section{Área de Estudio}

El área de estudio corresponde a la parte central y occidental de la Región Metropolitana, perteneciente a la cuenca del río Maipo, Mapocho y Puangue y los sistemas de esteros efluentes de la zona de Melipilla y San Pedro, hasta María Pinto. Esta área abarca prácticamente en su totalidad los sectores de montaña de la Cordillera de la Costa de la cuenca del Maipo, con una fuerte presencia de rocas graníticas, en el borde occidental y sur, como de la presencia de cobertura de cineritas. Destaca también la presencia de los valles intermontanos de Cholqui, Popeta, Puangue.

Se distinguen dos sectores morfológicamente diferentes, la sección norte de la depresión de Santiago, con importantes formas depositacionales y la sur, más bien de ambiente de media y baja montaña. Se incluyen además las áreas cubiertas por ARAYA-VERGARA (1985), de la cuenca del río Mapocho (Fig. $1)$. 
Figura 1

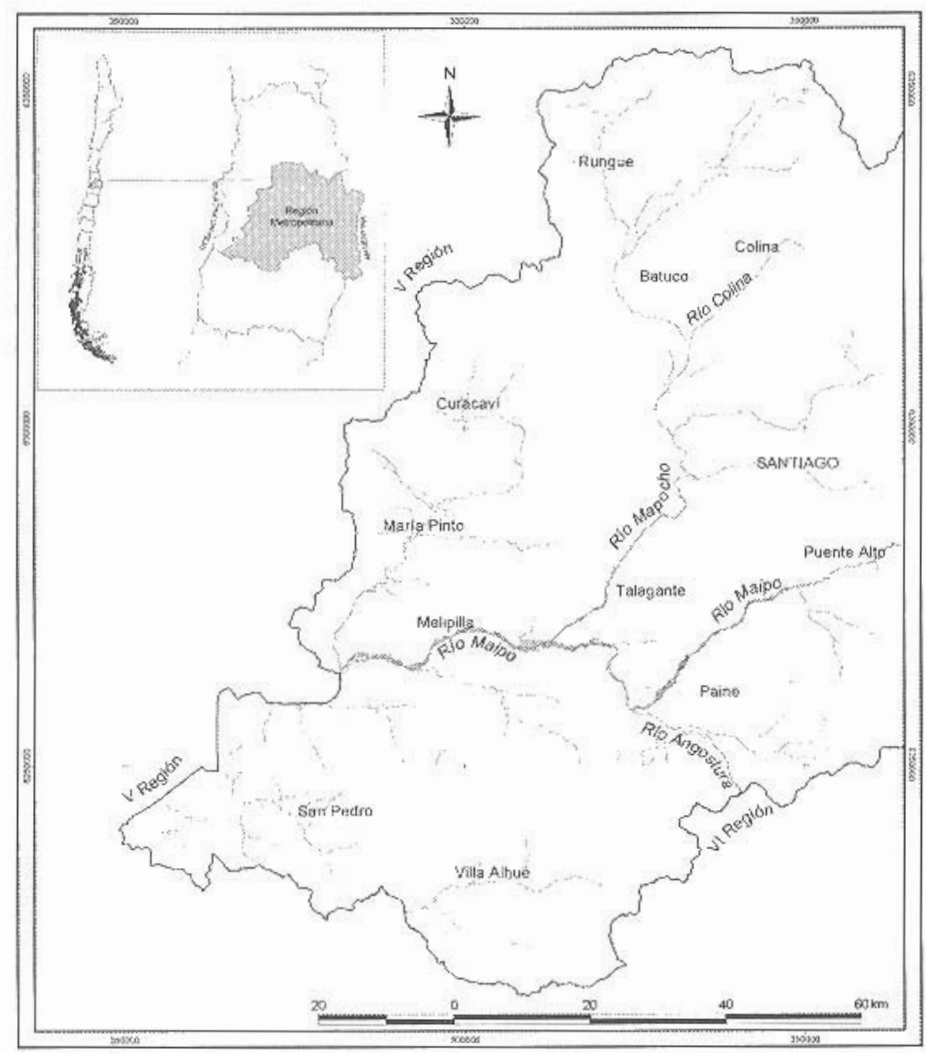

\section{RESULTADOS}

\section{Categorías de formas}

\section{Sistemas morfolitológicos de vertientes}

\section{Formas de excavación}

Las vertientes de excavación corresponden a grandes formas asociadas a procesos de denudación intensa en las partes culminantes de los cordones montañosos. Estas formas se presentan en Lipangue y Chicauma, y ya han sido descritas por ARAYA-VERGARA (1985). Estos sistemas, en sus partes basales presentan importantes sistemas de conos aluviales coalescentes, torrenciales, que suelen gradar hacia formas de glacis, sobre todo del tipo glacis de derrame. Sistemas de excavación fueron identificados bajo la forma de alvéolos en Cholqui y Alhué, modelados en sistemas de vertientes graníticas. Las partes culminantes de las vertientes de excavación presentan superficies culminantes que, según ARAYA-VERGARA (2000), corresponden a superficies de aplanamiento residual en los Cerros de Alhué. Similares características están presentes en Altos de Cantillana.

\section{Vertientes de influencia estructural}

Estos sistemas de vertientes tienen una amplia cobertura en la Región Metropolita y corresponden a todos aquellos flancos de valle modelados en estructuras monoclinales, sin diferenciar si se trata del borde consecuente (conformes), obsecuente (inversas o discordantes). La diferenciación antes mencionada no se realiza, en la medida que se considera que bajo las condiciones de media y baja montaña, la respuesta geodinámica de estos afloramientos rocosos es bastante similar. 
Para el ambiente de alta montaña, es recomendable realizar la distinción del estilo de plegamiento (ARAYA-VERGARA, 1985).

Se ha considerado además la inclusión del grupo taxonómico denominado como cordillera estilo eyectivo (Comunicación verbal, José Araya-Vergara 2007), que incluye el borde oriental de la depresión de Santiago. Su principal característica es el plegamiento con un radio de curvatura muy pequeño.

No obstante lo anterior, se consideró de fundamental importancia el distinguir la litología de los relieves en estructuras plegadas, a través de la consideración implícita de las formaciones geológicas. En este sentido se destacan las categorías de cobertura detrítico volcánica y calcáreas. Las primeras corresponden a sistemas de vertientes en rocas volcánicas de la Formación Veta Negra como a las formaciones Horqueta, Las Chilcas y Abanico, estas últimas de gran presencia en el área de estudio. Los sistemas de vertientes monoclinales modeladas en ambiente con predominio de estratos calcáreos corresponden al miembro inferior de la Formación Lo Prado.

\section{Vertientes modeladas en rocas graníticas}

La otra categoría de vertientes corresponde a aquellas modeladas en rocas graníticas, las que tienen una amplia distribución en el área de estudio, sobre todo en el borde S/E y Este. Este tipo de vertientes se encuentra muy bien desarrollado en San Pedro, María Pinto y Curacaví. El aspecto general de estos relieves corresponde claramente a una media y baja montaña del ambiente denominado secano costero interior, con relieves suavizados, de poca pendiente y vegetación del tipo estepa de Acacia caven.

ARAYA-VERGARA (2000) al tratar los relieves graníticos del coast range de Chile central, señala tres aspectos básicos observados: la presencia de superficies residuales, la meteorización profunda y los efectos de la tectónica. Tales aspectos para el área de estudio de la cuenca de Santiago, han sido reconocidos a través de los procesos de regolitización intensa y abundancia de grus, como también, la presencia de bloques en las vertientes y la acción de la erosión lineal.

El sector Altos de Cantillana es considerado como una superficie de erosión residual, similar a Cerros de Alhué, de la misma manera que la denominada superficie de Poca Pena, localizada en un piso altitudinal inferior, y separados por un sistema de fallas (SOTO et al., 2006). En este contexto destaca la presencia de sistemas de vertientes graníticas con alturas superiores a los 1.000 m.s.n.m., como Altos de Cantillana y Cerros de Alhué, y aquellos bajo esta cota, en San Pedro y Cholqui.

\section{Formas de glacis}

En cuanto a los glacis, estos han sido clasificados según ARAYA-VERGARA (1985), distinguiendo entre los coluviales, de derrame y de ahogamiento. El primer tipo corresponde a formas de base de vertiente ligadas a los procesos de arroyada. Son formas heredadas del Cuaternario, en las que se desarrollan procesos dinámicos actuales gracias a la acción de flujos de detritos canalizados por los talwegs preexistentes. Los glacis de derrame son aquellas formas depositacionales desarrolladas a partir del lavado de los sedimentos de calibre más fino desde los conos aluviales. A su vez, los glacis de ahogamiento corresponden en el caso del área de estudio al glacis de Colina, cuyo lavado de finos hacia las partes distales se ve entrampada por la presencia de relieves de montaña y del glacis de derrame de Lipangue, lo que genera condiciones de endorreísmo local y consecuente mal drenaje.

\section{Formas polifásicas de terrazas}

Corresponde a las formas polifásicas de ARAYA-VERGARA (1985), y que en este trabajo incorpora los diferentes niveles de terrazas encontrados según edades, período y época, extraídas de WALL et al. (1996) y de SELLÉS \& GANA (2001). 
La terraza holocénica corresponde al nivel más bajo, e incluye a las denominadas terraza $T^{\circ}$ y $T^{\prime}$. Estas terrazas presenta rodados frescos, sin suelos y una condición de inundación estacional, la primera y episódica, la segunda. La distinción de estos niveles en cuanto a la presencia y altura relativa de la $\mathrm{T}$ ' con respecto a las terrazas pleistocénicas es relevante para la comprensión de los fenómenos de crecidas fluviales. En este sentido, destaca la mayor complejidad de formas polifásicas de los llanos aluviales del sistema Mapocho-Maipo.

En la categoria de terraza aluvial pleistocénica se han agrupado los niveles de T1, T2 y remanentes de $\mathrm{T} 3$, sin hacer distinción entre ellas, pues ello requiere de método de radiación absoluta que transciende a los objetivos de esta investigación.

Finalmente, se distinguió una terraza aluvial indeterminada, que corresponde a dos situaciones especiales, como el sector de San Pedro, que corresponde morfológicamente a una terraza solevantada y antigua, con presencia de rodados en superficie, muy meteorizados y con suelos con alto contenido de arcillas. Su aspecto en terreno se asemeja más a sistemas de vertientes bajos y suavizados de coast range, que a terrazas aluviales propiamente tal. El otro sector, se localiza en Quilicura y Huechuraba y corresponde a una superficie de llano aluvial circunscrita al glacis de derrame y ahogamiento del río Mapocho y Colina.

\section{Formas de acumulación}

Esta categoría puede ser definida como Piedmont. Corresponde a los conos aluviales torrenciales y a los grandes conos aluviales, tal como fueron descritos por ARAYA-VERGARA (1985). Los primeros son formas menores coalescentes que estructuran un verdadero sistema, en que a esta escala de trabajo es difícil diferenciar con respecto a su origen aluvial o coluvial propiamente tal, pues ambos procesos coexisten en la génesis y dinámica actual de los sistemas basales de vertiente correlativos. Son frecuentes los aportes gravitacionales y los flujos de detritos en los taludes, generalmente estabilizados por vegetación, por lo que constituyen complejos basales coluvio-deyeccionales.

Respecto a las grandes unidades de acumulación, los conos regulares, se caracterizan por presentar una menor pendiente respecto a las anteriores, y constituyen prácticamente un continuum morfológico con las formas de terrazas aluviales del fondo de la depresión. Se distinguieron los conos regulares pleistocénicos, asociados principalmente a procesos deposicionales de las cuencas montañosas de los ríos Maipo y Mapocho. El cono regular de Isla de Maipo, ha sido considerado como holocénico, y estado asociado genéticamente a un reacomodo posterior de los materiales del cono de Buin.

\section{DISCUSIÓN}

La noción de sistemas para el tratamiento de las formas de las vertientes incorporada por ARAYA-VERGARA (1985) sigue siendo un concepto apropiado para los complejos geomorfológicos propios de los ambientes de cordillera y valle asociado.

La consideración explícita de las características geológicas como base para la identificación y mapeo de unidades geomorfológicas dinámica de gran extensión espacial, se ajusta a los preceptos metodológicos de PEÑA et al., (1997) y DESSENA et al., (2005) y CICCACCI et al., (2006) y complementa la determinación de la condición geodinámica del paisaje.

A partir de las características morfoestructurales y geodinámicas de la cuenca de Santiago se deduce la existencia de tres grandes secciones, a saber, la correspondiente a formas del piedmont en fondo de depresión junto al borde oriental andino; la sección norte, de depresión periférica (ARAYA-VERGARA, 1985) y la sección Este, S/E y Sur, en ambiente de coast range, denominación de ARAYAVERGARA (2000), sustentada en sus avances en relieves graníticos. Si bien a cada sección se asocian formas y procesos geodinámicos, es constante la predominancia de las formas 
heredadas del Cuaternario, como también la dinámica de procesos actuales, como la presencia de flujos de detritos en los sistemas de conos y talwegs, aporte gravitacional y erosión lineal en los sistemas de vertientes y conos, o bien desbordes fluviales.

No obstante lo anterior, es importante realizar la distinción entre las formas asociadas a flanco de valle andino, de aquellas correspondientes a relieves graníticos. En las primeras destacan las grandes formas aluviales de conos regulares y terrazas fluviales que conforman el fondo de la depresión.

En los relieves graníticos de la Cordillera de la Costa destaca la presencia de las superficies de erosión residual (ARAYA-VERGARA, 2000; SOTO et al., 2006), y los procesos de regolitización intensa, erosión, presencia de bloques en las vertientes y aporte de regolito hacia los talwegs. La presencia de la acción tectónica es importante en la medida que existen superficies residuales por sobre y bajo los 1.000 m.s.n.m, como también la parte del coast range, de la sección sureste.

\section{CONCLUSIONES}

Existen tres grandes secciones en la cuenca de Santiago, asociadas a la condición de flanco de valle andino, depresión periférica $\mathrm{y}$ de coast range, las que se distinguen claramente por las formas de acumulación y del piedmont, heredados del Cuaternario, y por los procesos dinámicos actuales. En las tres secciones predominan los flujos de detritos de carácter estacional, sobre todo en los sistemas de vertientes y en los conos torrenciales.

En la Cordillera de la Costa, en ambiente de coast range, predominan los procesos de erosión y la presencia de regolitización profunda. Existen superficies de erosión residual a diferentes altitudes. Los sistemas de acumulación son menos expresivos y las formas de glacis, reducidas, no pueden ser representadas a la escala del trabajo presente. Los sistemas de vertientes proveen abundante material detrítico y regolito a los talwegs, y presentan un aspecto más bien rexistático, a diferencia del flanco andino, que en el piso de media montaña comprendido por la carta geomorfológica, es más bien biostático.

\section{Agradecimientos}

Los autores agradecen al Profesor Titular. Sr. José Francisco Araya-Vergara por la revisión crítica del manuscrito y por sus valiosos aportes a esta publicación, fruto de su vasta trayectoria científica.

\section{Bibliografía}

ARAYA-VERGARA, J.F. 1985. Análisis de la carta geomorfológica de la cuenca del Mapocho. Inform. Geog. Chile, 32:31-44.

ARAYA-VERGARA, J.F. 1988. Toward a classification of slope systems. En 26th ${ }^{\mathrm{a}}$ Congress Intern. Geogr. Union. Sydney. Vol.1. 1:19 A2.

ARAYA-VERGARA, J.F. 1996. Primeras experiencias con una clasificación dinámica de vertientes de montaña. En I Taller Internacional de Geoecología de Montaña y Desarrollo Sustentable de los Andes del Sur. Santiago: Universidad de Chile-Unicef-The United Nations University, pp. 389-399.

CASTRO, C.P., M.V. SOTO., G. IGOR \& E. DELGADO. 2002. Análisis comparativo de los riesgos geomorfológicos de alta montaña. Valle del río Limari y del río Cachapoal. Anales Sociedad Chilena de Ciencias Geográficas, pp. 3-12.

CASTRO, C.P., M.V., SOTO \& C. CHAVEZ. 2003. Aporte moderno de masa y su implicancia en la definición de niveles de riesgo natural. Tiempo y Espacio, 13: 185-199.

CHAVEZ, C. 2005. Amenazas naturales en media y baja montaña asociados al corredor de comercio Las Leñas, VI Región del Libertador Bernardo O'Higgins. Santiago: Memoria para optar al título de Geografo, Facultad de Arquitectura y Urbanismo, Universidad de Chile. Inédito. 
CICCACCI, S., M. DEL MONTE., L. PALMIERI. \& M.C. SALVATORE (2006) . Entità dei processi di denudazione e variación morfologiche resentí nell'area di Radocofani (Toscana Meridionales, Italia). En: Giuliano Rodolfo (Ed). Erosione hídrica in ambiente mediterraneo: valutazione directa e indirecta in areee sperimentali e bacini idrografici.Istituto Geografico Militare, Scuola Supoeriore di Geografia. Brigati-Genova. pp. 29-64

DESSENA , M.A., F. FANTOLA., G. RODOLFI. \& S. VACCA. (2005) La carta delle unità omogenee di territorio (land units) del sistema idrografico Flumendosa-Muralgia. En: Rodolfi, G., Dessena, M.A. \& Vacca, S. (Ed). Il sistema idrografico Flumendosa-Muralgia: le acque, i suoli e i processi erosivi. Brigati-Genova. pp. 47-68.

FERRANDO, F. 1994. La cuenca Andina del río Mapocho: Determinación de su estado de equilibrio morfodinámico. Informe final Fondecyt 1000-91, Inédito.

PEÑA. J.L., F. PELLICER CORELLANO., J. CÍA. CHUECA \& A. JULIÁN ANDRÉS. 1997. Leyendas para mapas geomorfológicos a escalas 1: $100.000 / 1$ : 200.000 y 1 : 25.000/1: 50.000. En: Peña Monné, J.L. (Ed.), Cartografía geomorfológica básica y aplicada. Geoformas Ediciones, Logroño, pp. 127-143.

SELLES, D. \& P. GANA. 2001. Geología del área Talagante-San Francisco de Mostazal.
Escala 1:100.000. Santiago: Servicio Nacional de Geología y Minería. Serie Geología Básica, $n^{\circ} 74$.

SOTO, M.V. \& C.P. CASTRO. 2003a.. Dinámica de los sistemas de vertientes de alta montaña, cuenca del río Cachapoal, Chile. En X Congreso Geológico Chileno. Concepción: Universidad de Concepción, Departamento de Ciencias de la Tierra. Edición digital sin número de página.

SOTO, M.V. \& C.P. CASTRO. 2003b. Geodinámica moderna de vertientes periglaciares de altura, alta montaña de la cuenca del río Cachapoal, Chile. Invest. Geogr. Chile. 37: 1-19.

SOTO, M.V., CASTRO C.P. \& C. CHAVEZ. 2004. Caracterización geomorfológica del corredor de comercio Las Leñas, Valle del río Cachapoal, Andes de Chile Central. Revista Geográfica Norte Grande, 31: 85-98.

SOTO, M.V., C.P. CASTRO., G. RODOLFI,, M. MÄRKER \& R. PADILLA. 2006. Procesos geodinámicos actuales en ambiente de media y baja montaña de la cuenca del río Maipo, Región Metropolitana. Revista Geográfica Norte Grande, 35: 77-95.

WALL, R., P. GANA. \& A. GUTIÉRREZ. 1996. Mapa Geológico del área de San Antonio-Melipilla. Escala 1:100.000. Santiago: Servicio Nacional de Geología y Minería, Serie Geología Básica nº 2. 